\title{
A Spherically Symmetric Solution of the Maxwell-Einstein Equations
}

\author{
D. LOVELOCK* \\ Department of Mathematics, University of South Africa, Pretoria \\ Received January 11, 1967
}

Abstract. A spherically symmetric solution of the "already unified field theory" of RAINICH (i.e. of the source-free Maxwell-Einstein equations) is presented which represents a static massless charged particle. It is not equivalent to the ReissnerNordström solution with zero mass, although both metrics repel uncharged test particles.

\section{$\S 1$. Introduction}

In the absence of sources the Maxwell-Einstein equations are $^{1}$ (see [2])

$$
\left.\begin{array}{rl}
f_{i j ; k}+f_{k i ; j}+f_{j k ; i} & =0, \\
f_{; i}^{i j} & =0, \quad \text { and } \\
R_{i j}-\frac{1}{2} g_{i j} R & =2 f_{i h} f_{j}{ }^{h}-\frac{1}{2} g_{i j}\left(f_{r s} f^{r s}\right),
\end{array}\right\}
$$

where the electromagnetic field tensor $f_{i j}$ is defined in terms of the 4-potential $A_{i}$ by

$$
f_{i j}=A_{i ; j}-A_{j ; i} .
$$

It is well known [2], [3] that the system (1.1) is equivalent to the algebraic conditions

$$
\left.\begin{array}{rl}
R_{00} & \geqq 0, \\
R & =0, \quad \text { and } \\
R_{i j} R^{j}{ }_{k} & =\frac{1}{4} g_{i k}\left(R_{h j} R^{h j}\right),
\end{array}\right\}
$$

thereby giving rise to the so-called "already unified field theory" of RAINICH. In fact, if we are given a metric satisfying (1.3) we can construct the corresponding $f_{i j}$ in the following manner (for details see [3]). Bristol.

* On leave of absence from the Department of Mathematics, The University,

1 This note may in some respects be regarded as a continuation of [1] and we shall retain the same notation. 
We solve the equation

where

$$
\xi_{i j} \xi_{h k}=-\frac{1}{2} E_{\imath j h l}-\frac{1}{2}\left(R_{9 s} R^{r s}\right)^{-\frac{1}{2}} E_{i j l m} E_{h l i}^{l m},
$$

$$
E_{i j}^{h k}=\frac{1}{2}\left(-\delta_{i}^{h} R_{j}^{k}+\delta_{j}^{h} R_{i}^{k}-\delta_{j}^{k} R_{\imath}^{h}+\delta_{i}^{k} R_{j}^{h}\right),
$$

for $\xi_{i j}$ by setting $(i, j)=(h, k)$ and taking the root of $(1.4)$. We then construct the dual of $\xi_{i j}$ defined by ${ }^{2}$

$$
* \xi_{i j}=\frac{1}{2}(-g)^{\frac{1}{2}}[i j k l] g^{k h} g^{l r} \xi_{h r}
$$

and evaluate the quantity $\alpha$ by solving

$$
\frac{\partial \alpha}{\partial x^{i}}=(-g)^{\frac{1}{2}}[i j k l] R_{; s}^{j r} g^{s k} R_{r}^{l} /\left(R_{p q} R^{p q}\right) .
$$

Then the electromagnetic field tensor $f_{i j}$ is given by

$$
f_{i j}=\xi_{i j} \cos \alpha+* \xi_{i j} \sin \alpha .
$$

In this note we present a spherically symmetric solution of the system (1.3), and therefore (1.1), which corresponds to a massless charged particle at rest at the origin. It is not equivalent to the Reissner-Nordström solution with the mass term set equal to zero, although under this condition both metrics repel uncharged test particles (but by different amounts).

\section{$\S 2$. The Metric and the Electromagnetic Field}

In [1] the special line-element

$$
d s^{2}=\frac{a^{2}}{r^{2}}\left(c^{2} d t^{2}-d r^{2}-r^{2} d \theta^{2}-r^{2} \sin ^{2} \theta d \varphi^{2}\right),
$$

where $a$ is a constant, was discussed with particular reference to certain weakened vacuum field equations which have been proposed as alternatives to the Einstein vacuum field equations. In this section we shall attempt to answer the question which arises out of this, viz. to what physical field would the metric (2.1) correspond in the orthodox theory of General Relativity? As was pointed out in [1], (2.1) has a genuine singularity at $r=0$, which we interpret as a gravitational source (isolated mass), where $a$ is some quantity associated with the source. It is the interpretation of $a$ which is of interest.

In order to answer this question we recall some of the relations derived in [1] which are satisfied by (2.1). If

$$
x^{0}=c t, \quad x^{1}=r, \quad x^{2}=\vartheta, \quad x^{3}=\varphi,
$$

${ }^{2}[i j h k]$ is the Levi Civita symbol. It changes sign on interchange of any two adjacent indices and [0123] has the value unity. 
then

$$
\left.\begin{array}{rl}
R_{00} & =\frac{1}{r^{2}}, \\
R & =0, \quad \text { and } \\
R_{i j} R_{k}^{j} & =\frac{1}{4} g_{i k}\left(R_{h j} R^{h j}\right) .
\end{array}\right\}
$$

By comparing (2.2) with (1.3) we see that the metric (2.1) is a solution of the "already unified field theory" of RAINICH and thereby represents the interaction of a gravitational field with a source-free Maxwell electromagnetic field. So that we may interpret $a$ and see exactly what type of electromagnetic field is represented by (2.1), we have to evaluate $f_{i j}$. We first calculate $\xi_{i j}$ from (1.4). It is easily seen that the only nonvanishing $\xi_{i j}$ are

$$
\xi_{23}=-\xi_{32}=a \sin \vartheta,
$$

and the only non-zero duals of $\xi_{i j}$ are

$$
* \xi_{01}=-* \xi_{10}=\frac{a}{r^{2}} \text {. }
$$

Substitution of (2.3) and (2.4) in (1.7) gives the array

$$
\left(f_{i j}\right)=\left(\begin{array}{cccc}
0 & \frac{a}{r^{2}} \sin \alpha & 0 & 0 \\
-\frac{a}{r^{2}} \sin \alpha & 0 & 0 & 0 \\
0 & 0 & 0 & a \sin \vartheta \cos \alpha \\
0 & 0 & -a \sin \vartheta \cos \alpha & 0
\end{array}\right)
$$

where $\alpha$ is a solution of (1.6). However, since (see [1], equation (2.10))

we find

$$
R^{h 3} ; 1=0 \text {, }
$$

$$
\alpha=\text { constant }
$$

We choose

so that (2.5) becomes

$$
\alpha=\pi / 2,
$$

$$
\left(f_{i j}\right)=\left(\begin{array}{cccc}
0 & \frac{a}{r^{2}} & 0 & 0 \\
-\frac{a}{r^{2}} & 0 & 0 & 0 \\
0 & 0 & 0 & 0 \\
0 & 0 & 0 & 0
\end{array}\right)
$$

The components of the 4-potential $A_{i}$ (which are not unique) are easily derived from (2.6), one set being

$$
\left(A_{i}\right)=\left(-\frac{a}{r}, 0,0,0\right) .
$$


This 4-potential obviously satisfies the Lorentz condition

as well as the condition

$$
A_{; i}^{i}=0,
$$

$$
A_{i} A^{i}=1 \text {. }
$$

From (2.6) and (2.7) we thus interpret $a$ as the charge. Consequently (2.1) corresponds to a massless particle of charge a at rest at the origin for all time. Furthermore, (2.1) is not the Reissner-Nordström metric [3]

$$
\begin{aligned}
d s^{2}=(1 & \left.-\frac{2 m}{r}+\frac{e^{2}}{r^{2}}\right) c^{2} d t^{2}-\left(1-\frac{2 m}{r}+\frac{e^{2}}{r^{2}}\right)^{-1} d r^{2}- \\
& -r^{2} d \theta^{2}-r^{2} \sin ^{2} \theta d \varphi^{2}
\end{aligned}
$$

in a different coordinate system even for the case $m=0$, for in that case

whereas for $(2.1)$

$$
R^{i j} R_{i j}=4 e^{4} / r^{8},
$$

$$
R^{i j} R_{i j}=4 / a^{4}=\text { constant } .
$$

We can therefore conclude that there exists at least two distinct spherically symmetric metrics which correspond to a massless particle of charge a, viz. (2.10) with $m=0$ and $e=a$, and (2.1). Attempts have been made to generalize Birkhoff's theorem [4] (according to which every spherically symmetric solution of the vacuum field equations

$$
R_{i j}=0
$$

may be reduced, by a coordinate transformation, to the Schwarzschild metric) to the Maxwell-Einstein equations (1.1). It has been shown $[5,6,7]$ that any spherically symmetric solution of the Maxwell-Einstein equations must be static. However, the remarks made above show that it is not true that every spherically symmetric solution of (1.1) may be reduced, by a coordinate transformation, to the Reissner-Nordström metric.

If, for the case (2.1), we consider the trajectories of (uncharged) test particles we find that the equations of motion (for $\vartheta=\pi / 2, \dot{\varphi}=0$ ) may be written in the form (see [1], equation (4.9))

$$
r^{\prime 2}=c^{2}-b^{2} / r^{2},
$$

where a prime denotes differentiation with respect to $t$ and $b$ is a constant. However, in the Reissner-Nordström case (with $m=0$ ), the corresponding equations of motion are

$$
\dot{r}^{2}=h-e^{2} / r^{2},
$$

where a dot denotes differentiation with respect to $s$ and $h$ is a constant. The formal similarity between (2.11) and (2.12) is striking, but it should be stressed that the independent variables are $t$ and $s$ respectively. 
Nevertheless, both metrics predict that a massless charged particle will repel an uncharged test particle although by different amounts ${ }^{3}$.

Acknowledgements. I am very grateful to the staff of the University of South Africa, and in particular to Professor H. RuND, for the hospitality shown to me during my stay in Pretoria.

\section{Bibliography}

1. Lovelock, D.: Weakened field equations in general relativity admitting an 'unphysical' metric. Commun. math. Phys. 5, 205-214 (1967).

2. RaInich, G. Y.: Electrodynamics in the general relativity theory. Trans. Am. Math. Soc. 27, 106-130 (1925).

3. Wheeler, J. A.: Geometrodynamics. Topics in modern physics, Vol. 1. New York: Academic Press 1962.

4. Birkhoff, G. D.: Relativity and modern physics. Cambridge: Harvard University Press 1923.

5. Hoffmann, B.: On the spherically symmetric field in relativity. Quart. J. Math. 3, $226-237$ (1932).

6. - On the spherically symmetric field in relativity II. Quart. J. Math. 4, $179-183$ (1933).

7. - On the extension of Birkhoff's theorem to the case in which an electromagnetic field is present. Recent developments in general relativity, 279-282. London: Pergamon 1962.

3 A theorem analogous to that proved in the Appendix of [1], may also be proved for metrics of the form

$$
d s^{2}=\lambda c^{2} d t^{2}-\lambda^{-1} d r^{2}-r^{2} d \theta^{2}-r^{2} \sin ^{2} \theta d \varphi^{2},
$$

where $\lambda$ is a positive, strictly monotonically decreasing function of $r$ alone. 